\title{
Age-related activity patterns are moderated by diet in Queensland fruit flies Bactrocera tryoni
}

\author{
J O H N PRENTER ${ }^{1,2}$, CHR I S T O P HER W. W E L D O N 3 \\ and P H I L L P W. T A Y L O R ${ }^{1}$ \\ ${ }^{1}$ Department of Biological Sciences, Macquarie University, Sydney, Australia, ${ }^{2}$ Department of Science, St Mary’s University \\ College Belfast, Belfast, U.K. and ${ }^{3}$ Department of Zoology and Entomology, University of Pretoria, Hatfield, South Africa
}

\begin{abstract}
Life-history parameters and the fitness of tephritid flies are closely linked to diet. Studies of locomotor behaviour can provide insights to these links, although little is known about how locomotor behaviour is influenced by diet. In the present study, video recordings of Queensland fruit flies Bactrocera tryoni Froggatt (Diptera: Tephritidae) ('Q-flies') that are maintained individually in cages are used to determine how diet affects the activity patterns (flight, walking, grooming, inactivity) of males and females at ages ranging from 4 to 30 days. The frequency and total duration of activities over 10-min trials are affected by diet, age and sex. Supplementation of diet with hydrolysed yeast results in a higher frequency and duration of flight in flies of all ages and both sexes. The effect of diet on other activities varies with age. Q-flies fed sugar only increase walking frequency steadily from 4 to 30 days posteclosion, whereas flies fed sugar + yeast have higher walking frequencies at 4 and 10 days than flies fed sugar only, although they then exhibit a sharp decline at 30 days post-eclosion. The frequency and duration of inactivity remain consistent in flies fed sugar + yeast, whereas flies fed sugar only exhibit a marked increase in inactivity from 4 to 30 days post-eclosion. Compared with older flies, 4 day-old Q-flies fed sugar only spend considerably more time grooming. The potential of activity monitoring as a quality control test for flies that are mass-reared for use in sterile insect technique programmes is discussed.
\end{abstract}

Key words. Behaviour, diet, inactivity, locomotor activity, sugar, Tephritidae, yeast hydrolysate.

\section{Introduction}

Nutrition exerts powerful effects on the fitness of many insects (Raubenheimer et al., 2009), including tephritid flies. In many tephritid flies, a diet combining sugar with yeast hydrolysate, a rich source of carbohydrates, amino acids, sterols, minerals and vitamins, is found to enhance reproductive development, sexual performance, egg production and lifespan compared with a diet of sugar alone, and is commonly recommended for mass-rearing and pre-release provisioning (Yuval et al., 2002, 2007; Liedo et al., 2010). Because tephritid flies are important pests, and some species are reared in massive

Correspondence: John Prenter, St Mary's University College Belfast, 191 Falls Road, Belfast BT12 6FE, Northern Ireland, U.K. Tel.: +44 (0)2890 268314; e-mail: j.prenter@smucb.ac.uk numbers for release in sterile insect technique programmes, the focus of most nutrition research is on developing dietary regimes that promote rapid development and high reproductive activity. However, to understand the mechanisms by which diet affects these life-history parameters and fitness, we need to understand associated physiology and behaviour. Studies of unrestrained locomotor activity can provide useful insights because behaviour provides a window to physiological state.

Locomotor activity is a central facet of almost all animal behaviour (Martin, 2003) with established links to foraging, reproductive success and predator avoidance (Cobb et al., 1987; Oufiero \& Garland, 2007; Irschick et al., 2008). Being energetically demanding (Lehmann \& Schützner, 2010), locomotor activity is expected to interact with diet and associated physiological state, and this expectation is borne out in the limited data that are available for tephritid flies. For 
example, Zou et al. (2011) report that daily activity of the Mexican fruit fly Anastrepha ludens fed a sugar-only diet is higher than activity of those fed both sugar and yeast extract. However, regardless of diet, the daily total distance travelled by A. ludens declines to similar low levels during the second half of the lifespan (Zou et al., 2011).

The Queensland fruit fly Bactrocera tryoni Froggatt (Diptera: Tephritidae) has been the focus of substantial research into how the inclusion of yeast hydrolysate (and in particular amino acids) in the diet can promote rapid development, high levels of sexual activity and longevity (Taylor et al., 2011). The addition of even a small amount of yeast hydrolysate to a diet of sugar increases longevity (Pérez-Staples et al., 2007, 2008, 2009; Fanson et al., 2009; Fanson \& Taylor, 2012a, 2012b).

Despite the substantial attention afforded to the effects of diet on life-history parameters and mating performance, there are no direct studies available regarding how diet affects locomotor activity in $B$. tryoni. A recent study highlights the effects of mass-rearing and irradiation on general activity patterns (flight, walking, grooming, inactivity) in B. tryoni (Weldon et al., 2010). Mass-reared flies exhibit a marked reduction in overall activity compared with wild-type flies, and reproductive sterilization with gamma radiation (70-75 Gy) is found to induce a further reduction. Unlike many other insects (e.g. Drosophila melanogaster: Gatti et al., 2000; Hadj Belgacem \& Martin, 2002), there is no evidence for sex differences in the general activity of $B$. tryoni (Weldon et al., 2010). Drawing on the techniques deployed by Weldon et al. (2010), the present study examines the effects of diet on locomotor activity patterns in male and female $B$. tryoni aged 4, 10 and 30 days post-adult eclosion. It is expected that activity patterns will vary with the underlying physiological state as a function of diet, sex and age.

\section{Materials and methods}

\section{Source and treatment of flies}

Bactrocera tryoni were obtained as pupae from New South Wales Department of Primary Industries mass-rearing facility [Elizabeth Macarthur Agricultural Institute (EMAI), Camden, NSW, Australia]. Further details of the mass-reared colony at EMAI and delivery methods are provided in Pérez-Staples et al. (2009) and Weldon et al. (2010).

At Macquarie University, B. tryoni were maintained in a controlled environment room under a LD $14: 10 \mathrm{~h}$ photocycle at $24-26{ }^{\circ} \mathrm{C}$ and $60-70 \%$ relative humidity. The illumination regimen consisted of $12 \mathrm{~h}$ of full-intensity lighting and simulated dawn and dusk periods in which lights were stepped on and off in four stages over the course of $1 \mathrm{~h}$. Three replicate cohorts of flies from different production periods were used to complete the study.

Adult flies were allowed to emerge from pupae into 5-L ventilated plastic cages $(24 \times 15 \times 15 \mathrm{~cm})$ in the laboratory. The flies were sorted by sex on the day of eclosion, housed in separate 5-L cages (70 flies per cage) and assigned to one of two diet treatments: dry granular cane sugar only, or sugar + yeast (yeast hydrolysate enzymatic, MP Biomedicals, Aurora, Ohio), which was provided in separate dishes to allow self-selection of intake. Flies were provided ad libitum access to water and food.

Locomotor activity of female and male $B$. tryoni with a diet of sugar only or sugar supplemented with yeast hydrolysate ('sugar + yeast') was tested at three ages that correspond with recognized stages of sexual development for $B$. tryoni of the tested strain when provided a sugar + yeast diet: 4,10 and 30 days after adult eclosion. At 4 days after adult eclosion, both female and male $B$. tryoni are still reproductively immature (Meats et al., 2004; Pérez-Staples et al., 2007). By 10 days after adult eclosion, both females and males are reproductively mature (Pérez-Staples et al., 2007). An age of 30 days after adult eclosion is close to the mean longevity of female and male $B$. tryoni when held in single-sex cages (Fanson \& Taylor, 2012a). When provided sugar-only diets, B. tryoni exhibit low levels of reproductive maturity even after 30 days, and this is especially evident for females (Pérez-Staples et al., 2007, 2011; Prabhu et al., 2008).

\section{Activity assays}

Individual flies were removed from group holding cages and placed in 370-mL clear polypropylene containers (height $47 \mathrm{~mm}$, diameter $119 \mathrm{~mm}$; Chanrol C10, Chanrol Pty. Ltd, Australia), which were fitted with a base of filter paper to provide contrast, and covered with the lid of a clear plastic petri dish (diameter $140 \mathrm{~mm}$ ). After a 2-min acclimation period, video recordings were made in MPEG-4 format of the activity of individual flies for $10 \mathrm{~min}$ periods using a digital video camera (Canon XL1; Canon, Japan) connected through a USB TV tuner (Evolution TV, Model ETV02; Miglia Technology Ltd, U.K.) via an Apple MacBook laptop computer (Apple Inc., Cupertino, California) to an external hard-drive (Maxtor OneTouch III; Maxtor Corporation, Milpitras, California) for storage. Video recordings were made between 10.00 and $16.00 \mathrm{~h}$, with the camera positioned directly above the polypropylene containers, under fluorescent overhead lighting supplemented with two $60-\mathrm{W}$ full spectrum bulbs positioned $500 \mathrm{~mm}$ above the plastic containers.

For each replicate, the activity of eight males and eight females from each diet group (sugar only, sugar + yeast) was recorded. The process was repeated at 4,10 and 30 days posteclosion, giving 96 recordings per replicate and a grand total of 288 observations over three replicates. Recordings were scored, in real time at a later date, for the frequency and duration of flights, walking, grooming and periods of inactivity using JWATCHER, version 1.0 (Blumstein \& Daniel, 2007).

\section{Statistical analysis}

Mutually exclusive activities (flight, walking, grooming, inactivity) were compared using multivariate analysis of variance (MANOVA), with diet, sex, age and time of day of recording (morning 10.00-12.00 h; early afternoon $12.00-14.00 \mathrm{~h}$; 

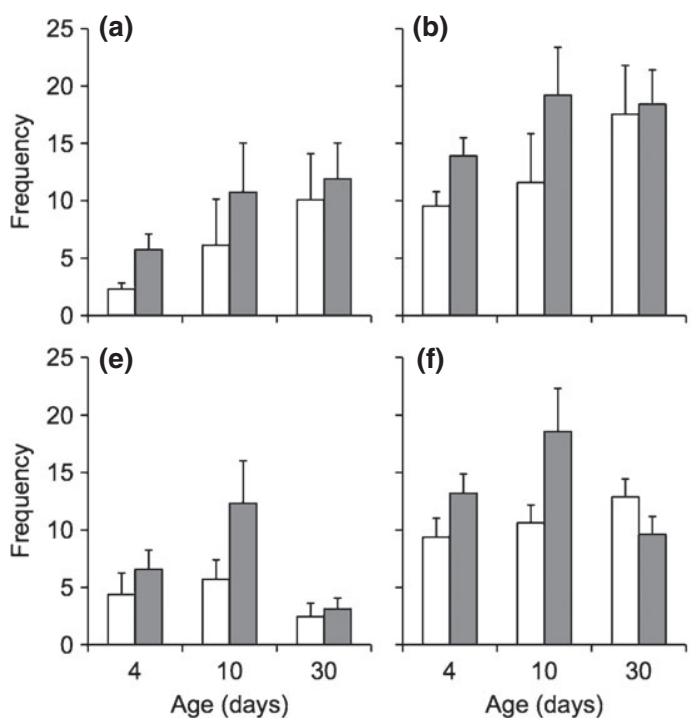

(c)
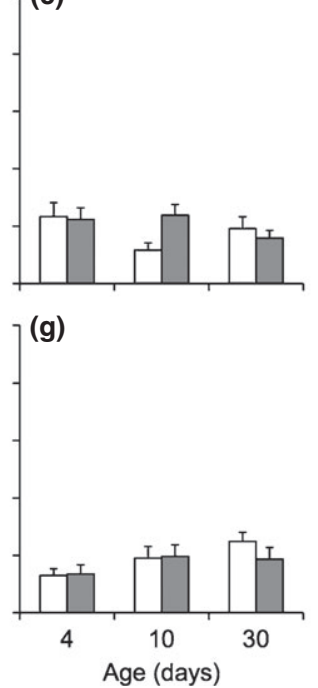

(d)

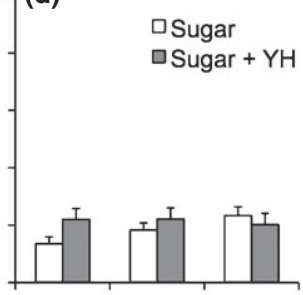

(h)

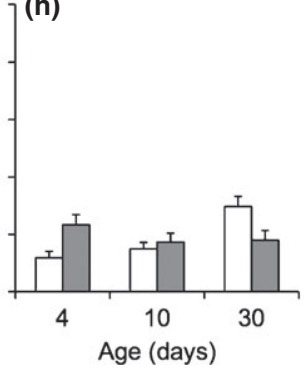

Fig. 1. Frequency of flying, walking, grooming and inactivity in relation to sex, age and diet in fertile mass-reared Bactrocera tryoni over a period of $10 \mathrm{~min}$. White bars represent sugar only diets; grey bars represent the sugar + yeast hydrolysate (YH) diet. Frequency of (a) flight, (b) walking, (c) grooming and (d) inactivity in female flies. Frequency of (e) flight, (f) walking, (g) grooming and (h) inactivity in male flies. Raw data are presented as the mean $\pm \mathrm{SE}$.

late afternoon $14.00-16.00 \mathrm{~h}$ ) as fixed effects. Interactions between diet and sex, diet and age, sex and age, diet and time of day, sex and time of day, and age and time of day were also examined. Before the analysis, frequencies and durations of activities were subjected to Box-Cox transformation (appropriate for zero inflated data; Hyndman \& Grunwald, 2000) to meet the assumptions of parametric analysis. Arcsine transformations have previously been advocated for this type of data (Fowler et al., 1998); however, the practice is no longer recommended (Warton \& Hui, 2011). The effect of replicate was discounted in preliminary analysis and this was omitted from the final model (Weldon et al., 2010), along with the nonsignificant three-way interactions $(P>0.1$; Sokal \& Rohlf, 1995). We further explored significant effects from MANOVAs in univariate analyses of variance and post-hoc tests of main effects (Tukey's honestly significant difference). All analyses were performed using STATISTICA, version 10 (StatSoft Inc., Tulsa, Oklahoma).

\section{Results and Discussion}

The frequency and the duration of bouts of different classes of activity in B. tryoni were affected by diet (sugar only, sugar + yeast), age (4, 10 or 30 days post-eclosion) and sex. There was, however, substantial variation in the effects across the treatment groups, with influential diet $\times$ age and age $\times$ sex interactions. MANOVA indicated that frequency of recorded behavioural states varied with the diet of $B$. tryoni, and the interactions of diet $\times$ age and age $\times \operatorname{sex}$ (Fig. 1 and Table 1). Similarly, the total duration spent by $B$. tryoni performing each activity over the $10 \mathrm{~min}$ period assessed varied with a diet $\times$ age interaction (Fig. 2 and Table 2). Strong effects
Table 1. Summary of multivariate analysis of variance on the effects of diet (sugar only, sugar + yeast hydrolysate), sex, age (4, 10, 30 days after eclosion), and time of day (ToD; morning $10.00-12.00 \mathrm{~h}$; early afternoon $12.00-14.00 \mathrm{~h}$; late afternoon $14.00-16.00 \mathrm{~h}$ ) on the frequency of locomotor activity in mass-reared Bactrocera tryoni over a period of $10 \mathrm{~min}$, as conducted on Box-Cox transformed data.

\begin{tabular}{llllrr}
\hline Source & d.f.num & d.f.den & Wilks' $\lambda$ & \multicolumn{1}{l}{$F$} & \multicolumn{1}{l}{$P$} \\
\hline Intercept & 4 & 265 & 0.1077 & 548.85 & $<0.0001$ \\
Diet & 4 & 265 & 0.9607 & 2.72 & 0.0306 \\
Sex & 4 & 265 & 0.9978 & 0.15 & 0.9657 \\
Age & 8 & 530 & 0.9706 & 1.00 & 0.4372 \\
Time of day & 8 & 530 & 0.9849 & 0.51 & 0.8528 \\
Diet $\times$ Sex & 4 & 265 & 0.9869 & 0.87 & 0.4796 \\
Diet $\times$ Age & 8 & 530 & 0.8927 & 3.87 & 0.0002 \\
Sex $\times$ Age & 8 & 530 & 0.9243 & 2.66 & 0.0072 \\
Diet $\times$ ToD & 8 & 530 & 0.9786 & 0.72 & 0.6729 \\
Sex $\times$ ToD & 8 & 530 & 0.9816 & 0.62 & 0.7624 \\
Age $\times$ ToD & 16 & 810 & 0.9693 & 0.52 & 0.9377 \\
\hline
\end{tabular}

of time of day on activity are reported for another tephritid fruit fly species (Anastrepha ludens; Zou et al., 2011), although such effects were absent from the present study (Tables 1 and 2), perhaps because fly activity was recorded outside the times of day when sexual advertisement, courtship and mating occur.

\section{Diet and age}

Diet had a significant effect on the frequency (Table 3) and duration (Table 4) of flight. The frequency and duration of flight tended to be higher in flies fed sugar + yeast 

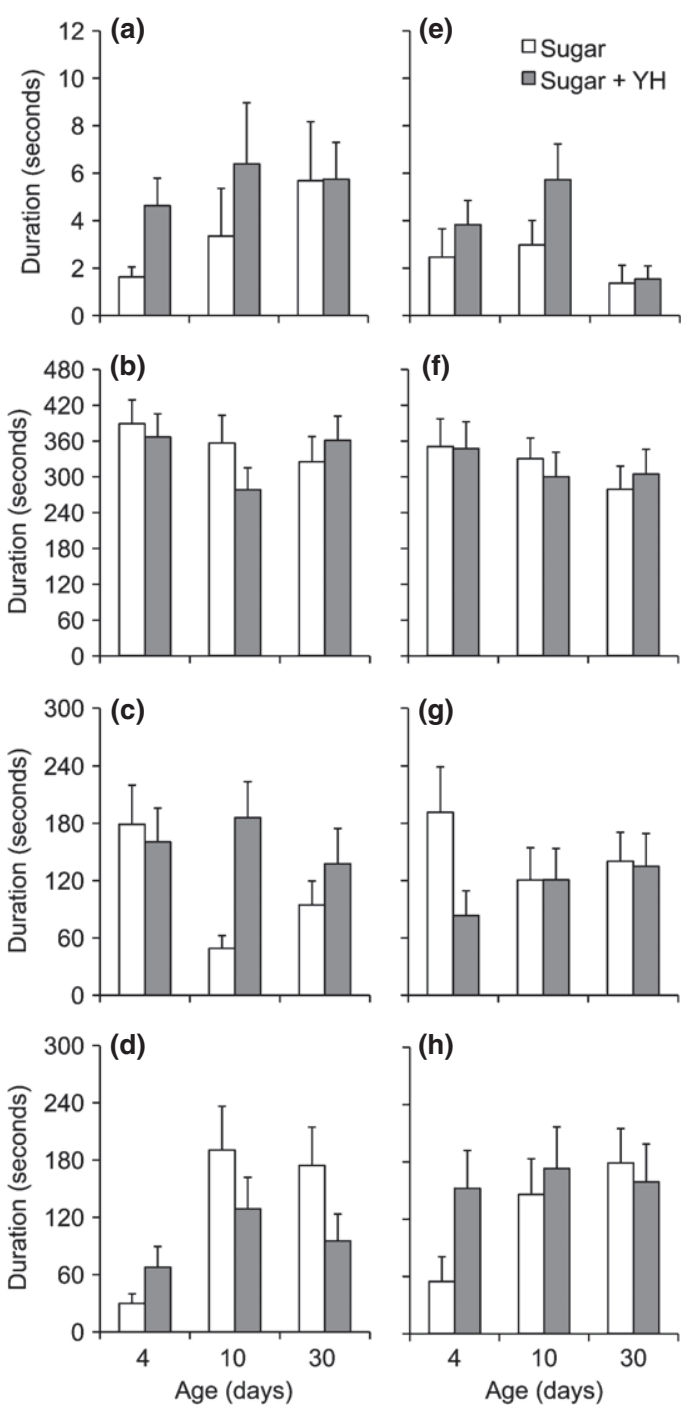

Fig. 2. Total duration of flying, walking, grooming and inactivity in relation to sex, age and diet in mass-reared Bactrocera tryoni over a period of $10 \mathrm{~min}$. White bars represent sugar only diets; grey bars represent the sugar + yeast hydrolysate $(\mathrm{YH})$ diet. Duration of (a) flight, (b) walking, (c) grooming and (d) inactivity in female flies. Duration of (e) flight, (f) walking, (g) grooming and (h) inactivity in male flies. Raw data are presented as the mean $\pm \mathrm{SE}$.

hydrolysate compared with flies fed sugar only (Fig. 1). Being an energetically demanding activity (Lehmann \& Schützner, 2010), it is not surprising that flight activity tended to be greater in flies receiving sugar + yeast diets compared with flies receiving sugar only (Fig. 1). The effects of diet on flight activity were similar for males and females of all ages, at all times of day, and so the physiological effects of yeast supplementation on flight activity would appear to be common across these contexts.

Diet also influenced patterns of other measured activities but, in each case, the effect of diet varied with age. The significant diet $\times$ age interaction identified by MANOVA for both the frequency and duration of bouts of activity was driven
Table 2. Summary of multivariate analysis of variance on the effects of diet (sugar only, sugar + yeast hydrolysate), sex, age (4, 10 and 30 days after eclosion), and time of day (ToD; morning 10.00-12.00 h; early afternoon $12.00-14.00 \mathrm{~h}$; late afternoon $14.00-16.00 \mathrm{~h}$ ) on the duration of locomotor activities (flight, walking, grooming, inactivity) in mass-reared Bactrocera tryoni over a period of $10 \mathrm{~min}$, as conducted on Box-Cox transformed data.

\begin{tabular}{llllrr}
\hline Source & d.f.num & d.f.den & Wilks' $\lambda$ & \multicolumn{1}{l}{$F$} & \multicolumn{1}{c}{$P$} \\
\hline Intercept & 4 & 265 & 0.0328 & 1955.33 & $<0.0001$ \\
Diet & 4 & 265 & 0.9432 & 3.99 & 0.0037 \\
Sex & 4 & 265 & 0.9940 & 0.40 & 0.8108 \\
Age & 8 & 530 & 0.9639 & 1.23 & 0.2802 \\
Time of day & 8 & 530 & 0.9896 & 0.35 & 0.9468 \\
Diet $\times$ Sex & 4 & 265 & 0.9829 & 1.15 & 0.3324 \\
Diet $\times$ Age & 8 & 530 & 0.9181 & 2.89 & 0.0037 \\
Sex $\times$ Age & 8 & 530 & 0.9465 & 1.85 & 0.0665 \\
Diet $\times$ ToD & 8 & 530 & 0.9740 & 0.88 & 0.5336 \\
Sex $\times$ ToD & 8 & 530 & 0.9683 & 1.08 & 0.3786 \\
Age $\times$ ToD & 16 & 810 & 0.9560 & 0.75 & 0.7407 \\
\hline
\end{tabular}

[Correction added on 19 June 2013, after first online publication: $P$ value for Intercept was changed from 0.0000 to $<0.0001$.]

by variation in the frequency of walking and inactivity (Table 3 ) and the duration of grooming and inactivity (Table 4), respectively. Flies fed sugar only increased walking frequency steadily from 4 days through to 30 days post-eclosion (Fig. 3a). By contrast, flies fed sugar + yeast walked more often than flies fed sugar only at 4 and 10 days of age but then declined in walking frequency at 30 days post-eclosion. These changes in walking frequency were not matched by significant changes in walking duration (Table 4), which could indicate an overall tendency for shorter bouts of walking in flies that walked more often.

The frequency (Fig. 3b) and duration (Fig. 4b) of inactivity was consistent across ages for flies fed sugar + yeast. By contrast, flies fed sugar only showed infrequent bouts of inactivity, spending little time inactive when 4 days posteclosion but this increased markedly at 10 and 30 days posteclosion. Although still reproductively immature, development of flies fed sugar + yeast is well under way at 4 days posteclosion. The development of flies fed sugar only is greatly delayed in comparison, although some flies on this diet do eventually exhibit some gonadal development (Pérez-Staples et al., 2011; Weldon \& Taylor, 2011) and sexual activity (Pérez-Staples et al., 2011) and so, to some extent, the increase in inactivity of these flies at 10 and 30 days post-eclosion might reflect delayed effects of development. However, as time passes, it is likely that flies suffered increasingly from deleterious effects of malnutrition and this might substantially explain the increased prevalence of inactivity. There is recent evidence of compensatory feeding in B. tryoni (Fanson et al., 2009; Fanson \& Taylor, 2012a, 2012b). This offers a credible explanation for both low levels of inactivity in young flies (4 days post-eclosion) and increased activity in older (30 days post-eclosion) flies fed sugar only because flies search for food with different nutritional composition to obtain essential nutrients that are lacking in a sugar-only diet.

The duration of grooming activity was also consistent across ages for flies fed sugar + yeast, whereas flies fed sugar only 
Table 3. Summary of univariate analysis of variance on the effects of diet (sugar only, sugar + yeast hydrolysate), sex, age (4, 10, 30 days after eclosion), and time of day (ToD; morning 10.00-12.00 h; early afternoon 12.00-14.00 h; late afternoon 14.00-16.00 h) on the frequency of locomotor activity in mass-reared Bactrocera tryoni over a period of $10 \mathrm{~min}$, as conducted on Box-Cox transformed data.

\begin{tabular}{|c|c|c|c|c|c|c|c|c|c|}
\hline & \multirow[b]{2}{*}{ d.f. } & \multicolumn{2}{|c|}{ Flight } & \multicolumn{2}{|c|}{ Walking } & \multicolumn{2}{|c|}{ Grooming } & \multicolumn{2}{|c|}{ Inactivity } \\
\hline & & $F$ & $P$ & $F$ & $P$ & $F$ & $P$ & $F$ & $P$ \\
\hline Intercept & 1 & 265.92 & $<0.0001$ & 1439.65 & $<0.0001$ & 581.32 & $<0.0001$ & 894.28 & $<0.0001$ \\
\hline Diet & 1 & 9.92 & 0.0018 & 9.03 & 0.0029 & 0.57 & 0.4491 & 0.19 & 0.6624 \\
\hline Sex & 1 & 0.21 & 0.6489 & 0.42 & 0.5161 & 0.29 & 0.5880 & 0.09 & 0.7613 \\
\hline Age & 2 & 0.08 & 0.9222 & 1.41 & 0.2466 & 0.42 & 0.6584 & 1.86 & 0.1574 \\
\hline ToD & 2 & 0.07 & 0.9300 & 0.13 & 0.8822 & 0.45 & 0.6360 & 1.31 & 0.2724 \\
\hline Diet $\times$ Sex & 1 & 0.20 & 0.6517 & 1.89 & 0.1705 & 2.57 & 0.1099 & 0.11 & 0.7400 \\
\hline Diet $\times$ Age & 2 & 0.17 & 0.8463 & 4.22 & 0.0157 & 2.64 & 0.0732 & 8.91 & 0.0002 \\
\hline Sex $\times$ Age & 2 & 5.15 & 0.0064 & 1.46 & 0.2341 & 3.62 & 0.0280 & 0.59 & 0.5561 \\
\hline Diet $\times$ ToD & 2 & 0.42 & 0.6594 & 0.36 & 0.7016 & 1.68 & 0.1874 & 0.31 & 0.7341 \\
\hline Sex $\times$ ToD & 2 & 0.92 & 0.3983 & 1.59 & 0.2065 & 0.15 & 0.8641 & 0.33 & 0.7219 \\
\hline Age $\times$ ToD & 4 & 0.68 & 0.6069 & 0.45 & 0.7758 & 0.35 & 0.8412 & 0.55 & 0.6978 \\
\hline Error & 268 & & & & & & & & \\
\hline Total & 287 & & & & & & & & \\
\hline
\end{tabular}

[Correction added on 19 June 2013, after first online publication: $P$ value for Diet $\times$ Age was changed from 0.00018 to 0.0002.]

Table 4. Summary of univariate analysis of variance on the effects of diet (sugar only, sugar + yeast hydrolysate), sex, age (4, 10, 30 days after eclosion), and time of day (ToD; morning 10.00-12.00 h; early afternoon 12.00-14.00 h; late afternoon 14.00-16.00 h) on the duration of locomotor activities in mass-reared Bactrocera tryoni during a period of $10 \mathrm{~min}$, as conducted on Box-Cox transformed data.

\begin{tabular}{|c|c|c|c|c|c|c|c|c|c|}
\hline & \multirow[b]{2}{*}{ d.f. } & \multicolumn{2}{|c|}{ Flight } & \multicolumn{2}{|c|}{ Walking } & \multicolumn{2}{|c|}{ Grooming } & \multicolumn{2}{|c|}{ Inactivity } \\
\hline & & $F$ & $P$ & $F$ & $P$ & $F$ & $P$ & $F$ & $P$ \\
\hline Intercept & 1 & 214.56 & $<0.0001$ & 860.98 & $<0.0001$ & 567.61 & $<0.0001$ & 742.60 & $<0.0001$ \\
\hline Diet & 1 & 11.65 & 0.0007 & 0.03 & 0.8666 & 0.35 & 0.5528 & 0.09 & 0.7614 \\
\hline Sex & 1 & 0.52 & 0.4707 & 0.34 & 0.5612 & 0.37 & 0.5412 & 0.32 & 0.5748 \\
\hline Age & 2 & 0.17 & 0.8439 & 1.07 & 0.3437 & 0.17 & 0.8415 & 3.32 & 0.0375 \\
\hline ToD & 2 & 0.32 & 0.7287 & 0.16 & 0.8562 & 0.16 & 0.8500 & 0.64 & 0.5303 \\
\hline Diet $\times$ Sex & 1 & 0.39 & 0.5333 & 0.09 & 0.7587 & 3.57 & 0.0600 & 0.60 & 0.4386 \\
\hline Diet $\times$ Age & 2 & 0.30 & 0.7384 & 0.77 & 0.4639 & 3.89 & 0.0216 & 6.51 & 0.0017 \\
\hline Sex $\times$ Age & 2 & 4.53 & 0.0116 & 0.31 & 0.7310 & 1.44 & 0.2386 & 0.35 & 0.7073 \\
\hline Diet $\times$ ToD & 2 & 0.81 & 0.4467 & 1.33 & 0.2670 & 0.56 & 0.5701 & 0.84 & 0.4318 \\
\hline Sex $\times$ ToD & 2 & 0.47 & 0.6276 & 3.06 & 0.0484 & 0.73 & 0.4807 & 0.37 & 0.6910 \\
\hline Age $\times$ ToD & 4 & 1.00 & 0.4081 & 0.66 & 0.6180 & 0.88 & 0.4759 & 1.36 & 0.2466 \\
\hline Error & 268 & & & & & & & & \\
\hline Total & 287 & & & & & & & & \\
\hline
\end{tabular}

[Correction added on 19 June 2013, after first publication: Inactivity $P$ value for Diet $\times$ Age was changed from 0.00018 to 0.0002 .]

tended to spend more time grooming when 4 days posteclosion than when 10 or 30 days post-eclosion (Fig. 4a). The consistency in grooming activity of flies on the sugar + yeast diet at the different ages tested makes sense given the need for grooming is not expected to change with developmental stage. Patterns in flies fed sugar only may relate to physical rather than nutritional differences in the diet. Yeast hydrolysate is sticky, as are excreta of flies provided this diet, and so flies in cages containing this diet might be expected to maintain relatively high levels of grooming. The sugar-only diet, and the resulting excreta, is not nearly as sticky and this likely explains the lower levels of grooming at 10 and 30 days posteclosion. However, this does not explain the elevated levels of grooming in flies fed sugar only at 4 days post-eclosion. For flies on a sugar-only diet, the main source of nutrition other than sugar would be the sticky excreta of other flies. In the days immediately after eclosion, this excreta may contain some nutrients carried over from the pupal stage (Nestel et al., 2004). Although such nutrients might still be reasonably abundant at 4 days post-eclosion, they are likely depleted by 10 days. Hence, the elevated levels of grooming in the sugar-fed flies at 4 days post-eclosion might be a response to the presence of more nutritious and sticky excreta at this time and elevated rates of coprophagy.

\section{Sex and age}

The significant sex $\times$ age interaction on overall frequency of activities identified by MANOVA (Table 1) was driven by variation in frequency of flight and grooming (Fig. 5 and Table 3). Sex differences in the frequency of flight activity 

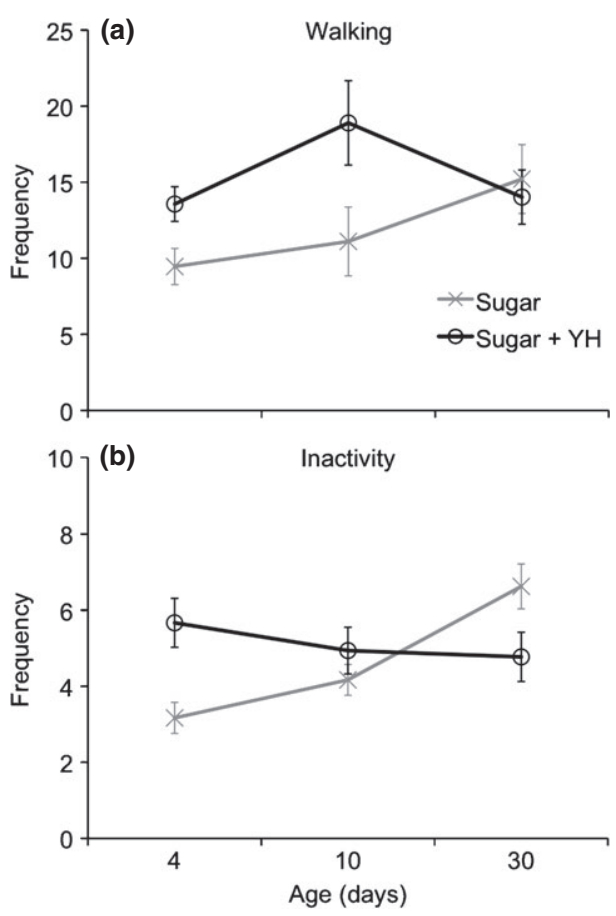

Fig. 3. Variation in the frequency of (a) walking and (b) inactivity of fertile mass-reared Bactrocera tryoni over a period of $10 \mathrm{~min}$ in response to diet and age. The diets provided to the flies comprised sugar and yeast hydrolysate (YH) or sugar only. Raw data are presented as the mean $\pm \mathrm{SE}$.

were evident only at the oldest age tested. Both sexes showed a pattern of increased flight activity from 4 to 10 days posteclosion but, whereas females then continued increasing flight activity levels to day 30 , males instead exhibited a substantial decline by day 30 (Fig. 5). Given that there was no evidence of differences between diet groups, we cannot ascribe this age effect to either maturation or ageing, both of which are linked closely to diet (Carey et al., 2008; Lee et al., 2008; Maklakov et al., 2008; Fanson et al., 2009; Fanson \& Taylor, 2012b).

Consistent with the previous study of Weldon et al. (2010), no sex differences in patterns of walking and inactivity of sexually immature $B$. tryoni were found (4 days post-eclosion; Tables 3 and 4). At this ontological stage, both sexes must forage for dietary resources needed for sexual development (Pérez-Staples et al., 2007); females require hydrolysed yeast in their diets to produce eggs and males also need it to reach peak sexual performance (Pérez-Staples et al., 2011). With similar selection pressures acting on females and males at this life stage, similar behavioural responses can be expected. Weldon et al. (2010) propose that high levels of grooming in mass-reared $B$. tryoni relative to their wild counterparts may reflect conditions associated with high population density. An increased prevalence of pathogens (Anderson \& May, 1981) could lead to selection for increased investment in activities related to resistance (Hochberg, 1991; Reeson et al., 1998). Considering the 4 day-old flies that fed sugar + yeast, a close match to the flies used in the study by Weldon et al . (2010), we observe low levels of grooming compared with the frequency
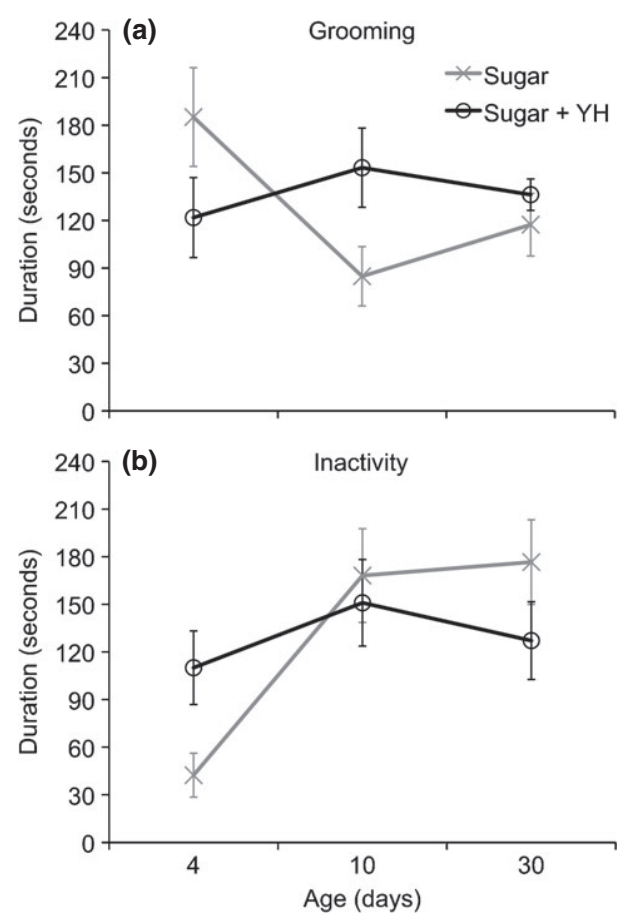

Fig. 4. Variation in the duration of (a) grooming and (b) inactivity of fertile mass-reared Bactrocera tryoni over a period of $10 \mathrm{~min}$ in relation to diet [sugar, sugar + yeast hydrolysate $(\mathrm{YH})$ ] and age. Raw data are presented as the mean $\pm \mathrm{SE}$.

and duration of walking, which is akin to that of wild flies reported by Weldon et al . (2010) for a similar age and the same diet. It is possible that variation in developmental conditions at the mass-rearing facility makes a considerable contribution to the behaviour of $B$. tryoni; many studies using the same mass-reared strain report substantial variation between cohorts in adult eclosion, flight ability, sex ratio and mortality under stress (Dominiak et al., 2002, 2008; Collins et al., 2008), and aspects of mating behaviour (Pérez-Staples et al ., 2007; Prabhu et al., 2008; Pérez-Staples et al., 2008).

\section{Inactivity and quality control}

Weldon et al. (2010) suggest that data on the frequency and duration of inactivity offers a simple but potentially sensitive and biologically relevant test of quality for massreared tephritids. Standardized quality control tests measuring the performance of mass reared tephritid flies are available, some of which assess locomotor performance, notably flight ability (FAO/IAEA/USD, 2003), startle (Boller et al., 1981) and predator avoidance (Hendrichs et al., 2007). Activity is generally related to health and vigour in insects (Martin, 2003) and is an important criterion used to assess sterile male performance (Hendrichs et al., 2007; Collins et al., 2008). The assessment of general activity lends itself readily to automation, using activity monitors of the type adopted for studies of Drosophila (Fernández et al., 1999; Martin 

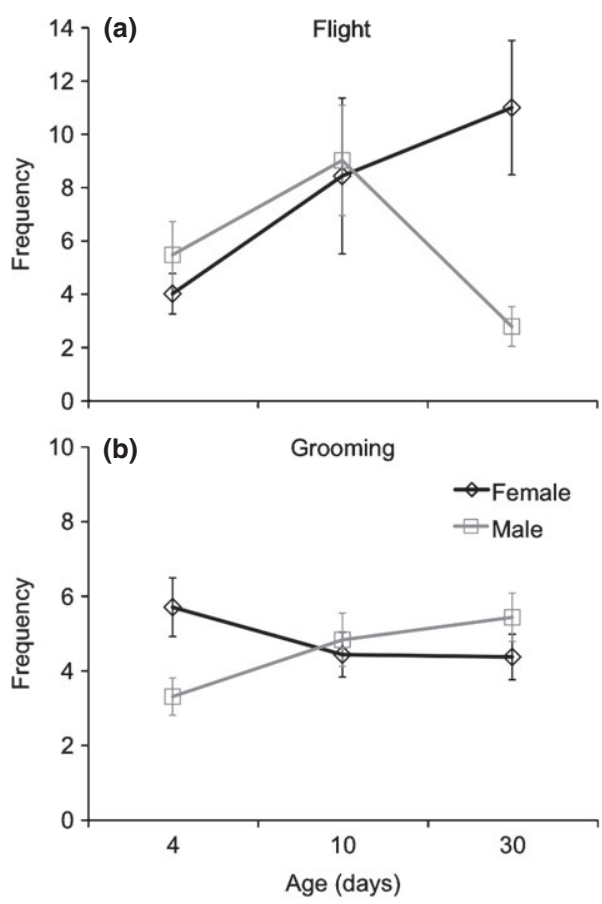

Fig. 5. Variation in the frequency of (a) flight and (b) grooming by fertile mass-reared Bactrocera tryoni over a period of $10 \mathrm{~min}$ in response to fly sex and age. Raw data are presented as the mean $\pm \mathrm{SE}$.

et al., 1999; Martin, 2003) to quantify bouts of activity and inactivity. Video-recordings, and especially automated systems, would mitigate the inter-individual and session variation in experimenter skills and observer effects that can easily affect startle and predator avoidance methods (Martin \& Bateson, 2007) and thus provide an effective, straighforward, highly repeatable alternative quality control measure in massrearing environments. This method might also be useful for providing an activity threshold for the selection of flies for control release. Weldon et al. (2010) report that activity levels of mass-reared domesticated flies are diminished relative to wild type flies, and that even further reductions are induced by irradiation for the induction of reproductive sterility. If the activity patterns of wild flies provide a quality benchmark defined by adaptation for field conditions, then we can interpret the lower activity seen in domesticated and irradiated flies as indicating diminished quality (i.e. fitness for field conditions). More active mass-reared flies would be more similar to wild flies and, by this standard, could be interpreted as being of higher quality than less active mass-reared flies. Further research is required to derive appropriate activity benchmarks if activity assays are to be developed for the assessment of fly quality in mass-rearing programmes.

\section{Acknowledgements}

We are grateful to staff at New South Wales Department of Primary Industries for providing pupae from the massrearing facility. We thank Rowan McGinley and Sam Collins for their assistance with the handling and rearing of flies and the recording of fly activity. This project was facilitated by Horticulture Australia Limited (HAL) in partnership with Australian Citrus Growers and was funded by the Citrus levy (project codes: CT05002 and CT07036). The Australian Government provides matched funding for all HAL R\&D activities.

\section{References}

Anderson, R.M. \& May, R.M. (1981) The population dynamics of microparasites and their invertebrate hosts. Philosophical Transactions of the Royal Society of London Series B, Biological Sciences, 291, 451-524.

Blumstein, D.T. \& Daniel, J.C. (2007) Quantifying Behaviour the JWatcher Way. Sinauer Associates Inc., Sunderland, Massachusetts.

Boller, E.F., Katsoyannos, B.I., Remund, U. \& Chambers, D.L. (1981) Measuring, monitoring and improving the quality of mass-reared Mediterranean fruit flies, Ceratitis canitata Wied 1. The RAPID quality control system for early warning. Zeitschrift für Angewandte Entomologie, 92, 67-83.

Carey, J.R., Harshman, L.G., Liedo, P. et al. (2008) Longevity-fertility trade-offs in the tephritid fruit fly, Anastrepha ludens, across dietary-restriction gradients. Aging Cell, 7, 470-477.

Cobb, M., Connolly, K. \& Burnet, B. (1987) The relationship between locomotor activity and courtship in the melanogaster species subgroup of Drosophila. Animal Behaviour, 35, 705-713.

Collins, S.R., Weldon, C., Banos, C. \& Taylor, P. (2008) Effects of irradiation dose rate on quality and sterility of Queensland fruit flies, Bactrocera tryoni (Froggatt). Journal of Applied Entomology, 132, $398-405$.

Dominiak, B.C., Sundaralingam, S., Jessup, A.J. \& Barchia, I.M. (2002) Pupal weight as a key indicator for quality of mass produced adult Queensland fruit fly Bactrocera tryoni (Froggatt) (Diptera: Tephritidae) in 1997/1998. General and Applied Entomology, 31, $17-24$

Dominiak, B.C., Sundaralingam, S., Jiang, L. et al. (2008) Production levels and life history traits of mass-reared Queensland fruit fly Bactrocera tryoni (Froggatt) (Diptera: Tephritidae) during 1999/2002 in Australia. Plant Protection Quarterly, 23, 131-135.

Fanson, B.G. \& Taylor, P.W. (2012a) Additive and interactive effects of nutrient classes on longevity, reproduction, and diet consumption in the Queensland fruit fly (Bactrocera tryoni). Journal of Insect Physiology, 58, 327-334.

Fanson, B.G. \& Taylor, P.W. (2012b) Protein: carbohydrate ratios explain lifespan patterns found in Queensland fruit fly on diets varying in yeast: sugar ratios. Age, 34, 1361-1368.

Fanson, B.G., Weldon, C.W., Pérez-Staples, D. et al . (2009) Nutrients, not caloric restriction, extend lifespan in Queensland fruit flies (Bactrocera tryoni). Aging Cell, 8, 514-523.

FAO/IAEA/USDA (2003) Manual for Product Quality Control and Shipping Procedures for Sterile Mass-Reared Tephritid Fruit Flies, Version 5.0. International Atomic Energy Agency, Austria.

Fernández, J.R., Grant, M.D., Tulli, N.M. et al . (1999) Differences in locomotor activity across the lifespan of Drosophila melanogaster. Experimental Gerontology, 34, 621-631.

Fowler, J., Cohn, L. \& Jarvis, P. (1998) Practical Statistics for Field Biologists, 2nd edn. John Wiley \& Sons, U.K.

Gatti, S., Ferveur, J.-F. \& Martin, J.-R. (2000) Genetic identification of neurons controlling a sexually dimorphic behaviour. Current Biology, 10, 667-670. 
Hadj Belgacem, Y. \& Martin, J.-R. (2002) Neuroendocrine control of a sexually dimorphic behavior by a few neurons of the pars intercerebralis in Drosophila. Proceedings of the National Academy of Sciences of the United States of America, 94, 10809-10812.

Hendrichs, M.A., Wornoayporn, V., Katsoyannos, B. \& Hendrichs, J. (2007) Quality control method to measure predator evasion in wild and mass-reared Mediterranean fruit flies (Diptera: Tephritidae). Florida Entomologist, 90, 64-70.

Hochberg, M.F. (1991) Viruses as costs to gregarious feeding behaviour in the Lepidoptera. Oikos, 61, 291-296.

Hyndman, R.J. \& Grunwald, G.K. (2000) Generalized additive modelling of mixed distribution Markov models with application to Melbourne's rainfall. Australian \& New Zealand Joumal of Statistics, 42, 145-158.

Irschick, D.J., Meyers, J.J., Husak, J.F. \& Le Galliard, J.-F. (2008) How does selection operate on whole organism functional performance capabilities? A review and synthesis. Evolutionary Ecology Research, 10, 177-196.

Lee, K.P., Simpson, S.J., Clissold, F.J. et al. (2008) Lifespan and reproduction in Drosophila: new insights from nutritional geometry. Proceedings of the Royal Society of London Series B, Biological Sciences, 273, 823-829.

Lehmann, F.-O. \& Schützner, P. (2010) The respiratory basis of locomotion in Drosophila. Journal of Insect Physiology, 56, 543-550.

Liedo, P., Orozco, D., Cruz-López, L. et al. (2010) Effect of postteneral diets on the performance of sterile Anastrepha ludens and Anastrepha obliqua fruit flies. Journal of Applied Entomology, in press. DOI:10.1111/j.1439-0418.2010.01568.x.

Maklakov, A.A., Simpson, S.J., Zajitschek, F. et al. (2008) Sexspecific fitness effects of nutrient intake on reproduction and lifespan. Current Biology, 18, 1062-1066.

Martin, J.-R. (2003) Locomotor activity: a complex behavioural trait to unravel. Behavioural Processes, 64, 145-160.

Martin, P. \& Bateson, P. (2007) Measuring Behaviour: An Introductory Guide, 3rd edn. Cambridge University Press, U.K.

Martin, J.-R., Ernst, R. \& Heisenberg, M. (1999) Temporal pattern of locomotor activity in Drosophila melanogaster. Joumal of Comparative Physiology, A, 184, 73-84.

Meats, A., Holmes, H.M. \& Kelly, G.L. (2004) Laboratory adaptation of Bactrocera tryoni (Diptera: Tephritidae) decreases mating age and increases protein consumption and number of eggs produced per milligram of protein. Bulletin of Entomological Research, 94, 517-524.

Nestel, D., Nemny-Lavy, E. \& Chang, C.L. (2004) Lipid and protein loads in pupating larvae and emerging adults as affected by the composition of Mediterranean fruit fly (Ceratitis capitata) meridic larval diets. Archives of Insect Biochemistry and Physiology, 56, 97-109.

Oufiero, C.E. \& Garland, T. Jr. (2007) Evaluating performance costs of sexually selected traits. Functional Ecology, 21, 676-689.

Pérez-Staples, D., Prabhu, V. \& Taylor, P.W. (2007) Post-teneral protein feeding enhances sexual performance of Queensland fruit flies. Physiological Entomology, 32, 225-232.
Pérez-Staples, D., Harmer, A.M.T., Collins, S. \& Taylor, P.W. (2008) Potential for pre-release diet supplements to increase the sexual performance and longevity of male Queensland fruit flies in field cages. Agricultural and Forest Entomology, 10, 255-262.

Pérez-Staples, D., Weldon, C.W., Smallridge, C. \& Taylor, P.W. (2009) Pre-lease feeding on yeast hydrolysate enhances sexual competitiveness of sterile male Queensland fruit flies in field cages, Entomologia Experimentalis et Applicata, 131, $159-166$.

Pérez-Staples, D., Weldon, C.W. \& Taylor, P.W. (2011) Sex differences in development response to yeast hydrolysate supplements in adult Queensland fruit fly. Entomologia Experimentalis et Applicata, 141, 103-113.

Prabhu, V., Pérez-Staples, D. \& Taylor, P.W. (2008) Protein: carbohydrate ratios promoting sexual activity and longevity of male Queensland fruit flies. Journal of Applied Entomology, 132, 575582.

Raubenheimer, D., Simpson, S.J. \& Mayntz, D. (2009) Nutrition, ecology and nutritional ecology: toward an integrated framework. Functional Ecology, 23, 4-16.

Reeson, A.F., Wilson, K., Gunn, A. et al. (1998) Baculovirus resistance in the noctuid Spodoptera exempta is phenotypically plastic and responds to population density. Proceedings of the Royal Society of London Series B: Biological Sciences, 265 , $1787-1791$.

Sokal, R.R. \& Rohlf, F.J. (1995) Biometry, 3rd edn. W.H. Freeman and Company, New York, New York.

Taylor, P.W., Pérez-Staples, D., Weldon, C.W. et al. (2011) Postteneral nutrition as an influence on reproductive development, sexual performance and longevity of Queensland fruit flies. Journal of Applied Entomology, in press. DOI:10.1111/j.14390418.2011.01644.x.

Warton, D.I. \& Hui, F.K.C. (2011) The arcsine is asinine: the analysis of proportions in ecology. Ecology, 92, 3-10.

Weldon, C.W. \& Taylor, P.W. (2011) Sexual development of wild and mass-reared male Queensland fruit flies in response to natural food sources. Entomologia Experimentalis et Applicata, 139, 17-24.

Weldon, C.W., Prenter, J. \& Taylor, P.W. (2010) Activity patterns of Queensland fruitflies (Bactrocera tryoni) are affected by both massrearing and sterilization. Physiological Entomology, 35, 148-153.

Yuval, B., Field, S.A., Blay, S. \& Taylor, P. (2002) Effects of postteneral nutrition on reproductive success of male Mediterranean fruit fly (Diptera: Tephritidae). Florida Entomologist, 85, 165-170.

Yuval, B., Maor, M., Levy, K. et al. (2007) Breakfast of champions or kiss of death? Survival and sexual performance of protein fed sterile Mediterranean fruit flies. Florida Entomologist, 90, $115-122$.

Zou, S., Liedo, P., Altamirano-Robles, L. et al. (2011) Recording lifetime behavior and movement in an invertebrate model. PLoS ONE, 6, e18151. 